\title{
Rapid liquid chromatography tandem mass spectrometry determination of rivaroxaban levels in human plasma for therapeutic drug monitoring
}

\author{
Andreea Varga ${ }^{1}$, Răzvan Constantin Șerban ${ }^{1, *}$, Daniela Lucia Muntean², \\ Cristina Maria Tătar ${ }^{3}$, Lenard Farczadi ${ }^{4}$, Ioan Tilea ${ }^{5}$ \\ ${ }^{1}$ University of Medicine and Pharmacy Tîrgu Mureș, Romania, \\ ${ }^{2}$ Analytical chemistry and Drug analysis, Department of Fundamental Pharmaceutical Sciences, \\ Faculty of Pharmacy, University of Medicine and Pharmacy Tîrgu Mureș, Romania, \\ ${ }^{3}$ Internal Medicine III, Dept. M3, Faculty of Medicine, University of Medicine and Pharmacy \\ Tîrgu Mureș, Romania, \\ ${ }^{4}$ Vim Spectrum SRL, 409 Calea Sighişoarei, Corunca, 547367, Romania, \\ ${ }^{5}$ Family Medicine, Dept. M3, Faculty of Medicine, University of Medicine and Pharmcy, Tîrgu Mureș, \\ Romania
}

\begin{abstract}
A rapid, sensitive, high-throughput liquid chromatography coupled with tandem mass spectrometry method for the quantification of rivaroxaban from human plasma has been developed and validated. For the analytical separation a Zorbax SB-C18 column with isocratic flow of mobile phase composed of $0.2 \%$ formic acid in water and acetonitril $(65: 35, V / V)$ with a flow rate of $1 \mathrm{~mL} / \mathrm{min}$ at a temperature of $45^{\circ} \mathrm{C}$ was used. Detection of rivaroxaban was performed using positive electrospray ionization and MS/MS mode (sum of $\mathrm{m} / \mathrm{z} 231.1 ; 289.2$ and 318.2 from $\mathrm{m} / \mathrm{z}$ 436.3). Plasma samples were prepared using single-step protein precipitation with methanol. Method validation was performed with regards to selectivity, linearity $(r>0.9927)$, within-run and between-run precision $(C V<13.1$ $\%)$ and accuracy (bias $<9.4 \%$ ) over a concentration range of $24.00-960.00 \mathrm{ng} / \mathrm{mL}$ plasma. Recovery was between $96.5-108.5 \%$ and the lower limit of quantification of rivaroxaban was $24.00 \mathrm{ng} / \mathrm{mL}$. The developed method is simple, rapid, and selective, requires small plasma sample volumes, and was successfully applied for therapeutic drug monitoring of rivaroxaban in treated patients.
\end{abstract}

Keywords: Anticoagulants, direct factor Xa inhibitors, rivaroxaban, LC-MS/MS, therapeutic drug monitoring.

Received: $6^{\text {th }}$ November 2016; Accepted: 17 th January 2017; Published: $7^{\text {th }}$ March 2017

* Corresponding author: Răzvan Constantin Șerban, University of Medicine and Pharmacy, Gh. Marinescu 38, Tg Mureș, Romania, e-mail: angiografieiii@yahoo.com 


\section{Introduction}

Thromboembolic events are amongst the most common causes of morbi-mortality worldwide [1]. Although highly efficient, the use of vitamin $\mathrm{K}$ antagonists (VKAs), the gold standard in thromboembolism prophylaxis for more than 60 years, is far from being ideal. During the past decade, two new classes of target-specific oral anticoagulants (NOACs) - direct thrombin inhibitors and direct factor Xa inhibitors - were introduced as an appealing alternative to VKAs. Large clinical trials have demonstrated that these new agents are at least as effective and are generally safer than the VKAs [2-5]. Moreover, the more predictable pharmacokinetics and pharmacodynamics of these new agents supported their use in fixed doses, without the need for routine laboratory monitoring [6].

Consensus seems to have been reached, however, that "circumstantial" laboratory testing may be required in a number of clinical scenarios, such as in NOAC-treated patients presenting with acute hemorrhagic or thrombotic events, or who may benefit from semiurgent surgery, if bleeding risk is thought to be acceptable, or in cases of suspected overdose [7].

The problem remains, however, that whereas the classic coagulation assays cannot provide accurate, quantitative measure of plasma drug concentration or anticoagulant effect, most of the novel tests validated for laboratory assessment of NOACs require high training, dedicated equipment, and are not routinely available in most laboratories.

Liquid chromatography coupled with tandem mass spectrometry (LC-MS/MS) is the most widely used method for therapeutic monitoring and bioavailability studies of NOACs, due to very good sensitivity and selectivity, as well as adequate accuracy and precision [8-13] although other methods, such as surface-assisted laser desorption/ionization mass spectrometry
(SALDI-MS), have also been described [9]. Several therapeutic drug monitoring [8] and pharmacokinetic studies $[10,11]$ of rivaroxaban that use LC-MS/MS for its determination in human plasma have been described in literature.

The aim of this study was to develop and validate a new, simple, rapid high throughput LC-MS/MS method for the determination of rivaroxaban human plasma levels for use in therapeutic drug monitoring.

The protocol of this research was approved by the Ethics Committee of the University of Medicine and Pharmacy of Tîrgu Mureș, Romania, were the study was conducted. Research has been performed in the accordance with standards of 1964 Helsinki declaration and its later amendments or comparable ethical standards. All participants included in the study provided written informed consent.

\section{Material and methods}

\section{Reagents}

Gradient grade for liquid chromatography methanol and acetonitril, as well as ammonium formate and formic acid of analytical purity were acquired from Merck KGaA (Darmstadt, Germany). Bidistilled deionized water was obtained from a Millipore Milli-Q Water purifying system (Milford, USA). Blank human plasma was supplied by the Cluj-Napoca Regional Blood Transfusion Center (Romania) and was obtained from healthy male and female volunteers.

\section{Apparatus}

The following equipment were used: Laborzentrifugen 204 Centrifuge (Sigma, Osterode am Harz, Germany); Analytical Plus and Precision Standard Balances (MettlerToledo, Greifensee, Switzerland); Vortex Genie 2 mixer (Scientific Industries, New York, USA); 
Elma Transsonic 700/H ultrasonic bath (Singen, Germany); Biohit Proline automatic pipettes (Biohit, Helsinki, Finland). LC-MS system used: Agilent 1100 HPLC system composed of G1316A Column Oven, G1329A Autosampler, G1312A Binary Pump, G1379A Degasser coupled with Agilent 1100 SL Ion Trap Mass Detector (Agilent Technologies, Santa Clara, USA).

\section{Chromatographic and spectrometric conditions}

Analytical separation was performed using a Zorbax SB-C18 (3.0 x 100mm, $3.5 \mu \mathrm{m})$ (Agilent Technologies) chromatographic column using isocratic flow of mobile phase composed of $0.2 \%$ formic acid in water and acetonitril $(65: 35, \mathrm{~V} / \mathrm{V})$ with a flow rate of $1 \mathrm{~mL} / \mathrm{min}$ and at temperature of $45^{\circ} \mathrm{C}$. Detection of rivaroxaban was performed using positive electrospray ionization (positive ionization, capillary voltage $4000 \mathrm{~V}$, nebulizer gas (nitrogen) pressure 60 psi, dry gas (nitrogen) temperature $350^{\circ} \mathrm{C}$, dry gas (nitrogen) flow $12 \mathrm{~L} /$ $\mathrm{min}$ ) and MS/MS monitoring mode (sum of $\mathrm{m} / \mathrm{z}$ $231.1 ; 289.2$ and 318.2 from $\mathrm{m} / \mathrm{z} 436.3$ ). The method run-time was 1.7 minutes.

\section{Standard solutions}

Rivaroxaban stock solution with a concentration of $80.00 \mu \mathrm{g} / \mathrm{mL}$ was prepared by dissolving the appropriate quantity of rivaroxaban in methanol. The stock solution was diluted with blank human plasma to prepare two working solutions with concentrations of $2.40 \mu \mathrm{g} / \mathrm{mL}$ and $0.24 \mu \mathrm{g} / \mathrm{mL}$, respectively. The working solutions were subsequently diluted with plasma for the preparation of calibration standards with concentrations of $24.00,48.00,96.00,192.00$, $384.00,576.00,768.00$ and $960.00 \mathrm{ng} / \mathrm{mL}$, and quality control (QC) plasma samples with concentrations of $48.00 \mathrm{ng} / \mathrm{mL}$ (lower), 192.00 $\mathrm{ng} / \mathrm{mL}$ (medium) and $576.00 \mathrm{ng} / \mathrm{mL}$ (higher). All plasma calibration standard and QC samples were stored at $-20^{\circ} \mathrm{C}$ until analysis.

\section{Sample preparation}

Plasma calibration standard solutions, QC plasma solutions, and volunteer plasma samples $(100 \mu \mathrm{L})$ were deproteinized with methanol (300 $\mu \mathrm{L})$ in propylene tubes, mixed using the vortexmixer (10 s) and centrifuged ( $3 \mathrm{~min}$ at 10,000 $\mathrm{rpm})$. The obtained supernatant was transferred to chromatography vials and $6 \mu \mathrm{L}$ sample volume injected into the LC-MS/MS system.

\section{Method validation}

The analytical method was evaluated regarding selectivity, specificity, within- and betweenday accuracy and precision, analyte recovery, according to international guidelines [14, 15]. For the evaluation of selectivity, chromatograms obtained from plasma samples spiked with rivaroxaban were compared to chromatograms of blank plasma samples. The QuantAnalysis (Brucker Daltonics, Germany) software was used to automatically calculate rivaroxaban concentrations based on chromatographic peak areas using external standard method.

Calibration curves were constructed from single calibration standards, linear model, using a weighting factor $1 / y^{2}$. For determination of within- and between-run precision (expressed as coefficient of variation, $\mathrm{CV} \%$ ) and accuracy (expressed as relative difference between obtained and theoretical concentration, Bias $\%)$ five different samples $(n=5)$ of each QC standard (low, medium, and high level, respectively) were analyzed on the same day and in five different days, respectively. The lower limit of quantification (LLOQ) of rivaroxaban $(24.00 \mathrm{ng} / \mathrm{mL})$ was studied and both within-run and between-run accuracy and precision were calculated using five different LLOQ samples for each. Recovery of rivaroxaban was measured by comparing responses of plasma samples spiked with rivaroxaban with standard solution prepared in mobile phase containing the same 
drug concentration and processed the same as plasma samples.

\section{Therapeutic drug monitoring of rivaroxaban}

The analytical method was used for therapeutic drug monitoring of rivaroxaban in 29 patients receiving single daily doses of $20 \mathrm{mg}$ drug. Two blood samples were taken from each patient, at treatment steady-state, just before a new rivaroxaban dose administration (Sample_1) and 3 hours after administration (Sample_2). A CoaguChek ${ }^{\circledR}$ XS System (Roche Diagnostics, USA) was used for International Normalized Ratio (INR) measurements simultaneously with blood sampling. Plasma samples were obtained after blood sample centrifugation for $6 \mathrm{~min}$ at 4,000 rpm. The rivaroxaban plasma levels were obtained by using the currently described analytical procedure and the normalized plasma levels by dividing the concentration to body weight. For statistical evaluation of results (Boxplot graphs, descriptive statistics, analysis of variance ANOVA), Phoenix software (Pharsight, SUA) was used.

\section{Results}

To obtain the best analyte ionisation efficiency in mass spectrometer (finally translated as method sensitivity), a screening of the main factors affecting drug ionisation yield was realised. The studied factors were: ionisation source type (electrospray (ESI) positive mode and atmospheric pressure chemical ionization (APCI) positive mode), the organic modifier used (methanol and acetonitrile), the aqueous phase used $(0.2 \%$ formic acid in water and $1 \mathrm{mM}$ ammonium formate in water). The same amount of rivaroxaban standard solution was injected in the chromatographic system prepared in each unique combination of the three factors studied ( 8 combinations) and the signal-to-noise ratio of the chromatographic peak obtained was calculated (data not shown). The best analyte response was obtained using ESI positive mode ionization and a mobile phase composed of $0.2 \%$ formic acid in water and acetonitrile $(65: 35, \mathrm{~V} / \mathrm{V})$. In the acidic mobile phase rivaroxaban easily accepts a proton generating the pseudo-molecular ion $[\mathrm{M}+\mathrm{H}]^{+}(\mathrm{m} / \mathrm{z}$ 436.3) (Figure 1). After collision induced dissociation in the mass spectrometer, this pseudo-molecular ion ( $\mathrm{m} / \mathrm{z} 436.3)$ produces fragments (Figure 2). The fragments having $\mathrm{m} / \mathrm{z}$ $231.1, \mathrm{~m} / \mathrm{z} 289.2$, and $\mathrm{m} / \mathrm{z} 318.2$ were chosen for the quantification of rivaroxaban.

The developed method was optimized to obtain chromatographic peaks with minimal tailing and short retention times for rivaroxaban.

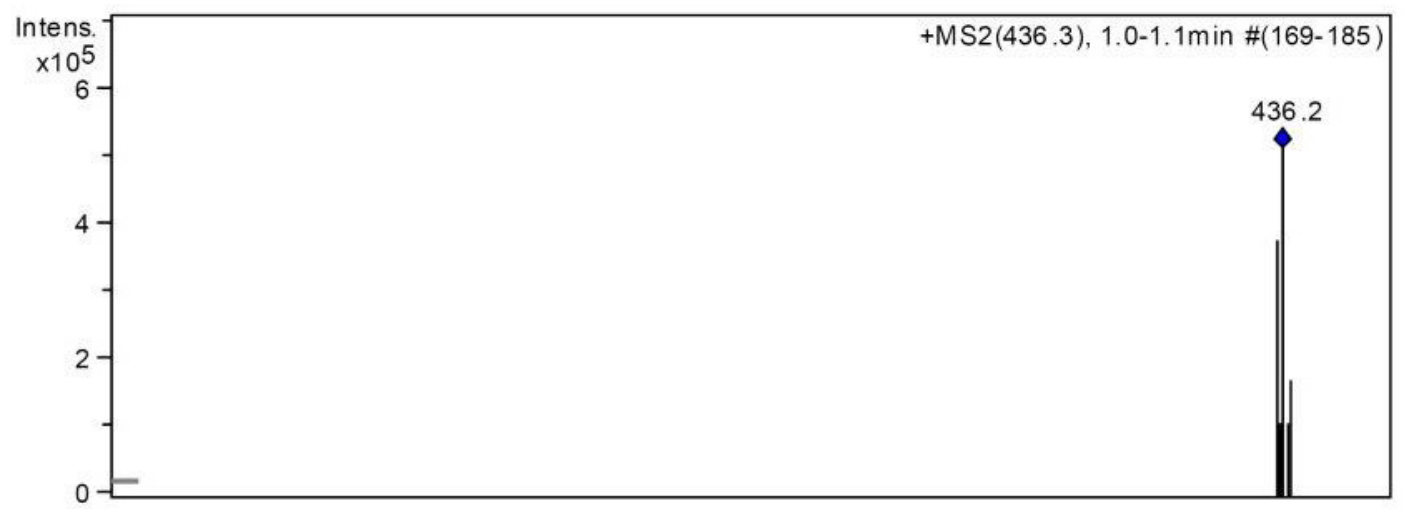

Figure 1. Non-reactive mass spectra of rivaroxaban 


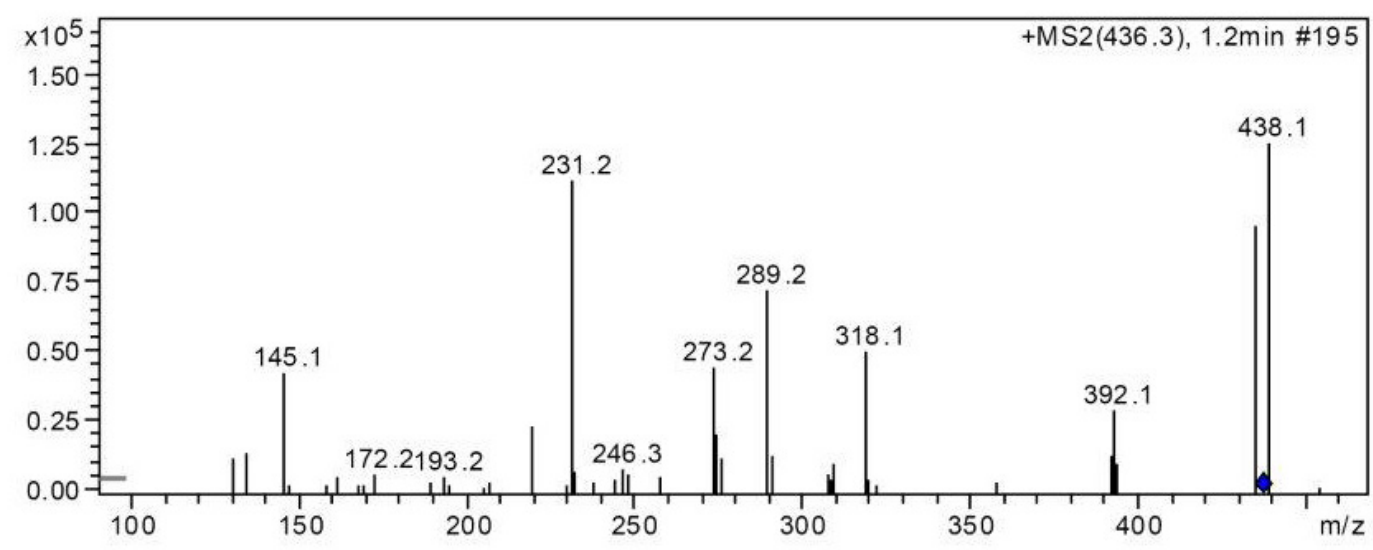

Figure 2. Collision induced dissociation (reactive) mass spectra

Table 1. Within-run accuracy (Bias), precision (CV) and recovery for rivaroxaban (C - concentration, $\mathrm{CV}$ - coefficient of variation, SD - standard deviation)

\begin{tabular}{ccccc}
\hline $\mathbf{c}_{\text {nominal }}(\mathbf{n g} / \mathbf{m l})$ & $\begin{array}{c}\text { Mean } \mathbf{c}_{\text {found }} \\
(\mathbf{n g} / \mathbf{m l}( \pm \text { SD) })\end{array}$ & Bias (\%) & CV (\%) & $\begin{array}{c}\text { Mean recovery } \\
(\% \text { ( } \mathbf{\text { SD }}))\end{array}$ \\
\hline $24.00(\mathrm{LLOQ})$ & $22.01(1.25)$ & -8.3 & 5.7 & $103.3(5.8)$ \\
\hline $48.00(\mathrm{QCA})$ & $46.27(3.95)$ & -3.6 & 8.5 & $96.5(6.9)$ \\
\hline $192.00(\mathrm{QCB})$ & $188.01(11.82)$ & -2.1 & 6.3 & $108.5(4.1)$ \\
\hline $576.00(\mathrm{QCC})$ & $521.87(9.83)$ & -9.4 & 1.9 & $102.4(7.8)$ \\
\hline
\end{tabular}

The best chromatographic result was achieved using a Zorbax SB-C18 $3.0 \times 100 \mathrm{~mm}, 3.5 \mu \mathrm{m}$ column thermostatted at $45{ }^{\circ} \mathrm{C}$ and a mobile phase mixture of $0.2 \%$ formic acid in water and acetonitrile $(65: 35, \mathrm{~V} / \mathrm{V})$ with isocratic flow and flow rate of $1 \mathrm{~mL} / \mathrm{min}$. Analyte retention time was $1.4 \mathrm{~min}$, while the sample run-time was $1.7 \mathrm{~min}$. The analysis of six blank plasma samples showed no interfering endogenous peaks at the retention time of rivaroxaban. Typical chromatograms of rivaroxaban in human plasma obtained during analytical method validation or in therapeutic drug monitoring study are presented in figure 3 .

All calibration curves had correlation coefficient greater than 0.9927 and were linear over the concentration range $24.00-960.00 \mathrm{ng} /$
mL plasma. Results for within-run and betweenrun precision, accuracy and recovery obtained during the validation are shown in Table I and Table II. The lower limit of quantification (LLOQ) was $24.00 \mathrm{ng} / \mathrm{mL}$ and had acceptable accuracy and precision results (Table $\mathbf{I}$ and Table II).

\section{Discussion}

Because of high inter- and intra-individual variability in the pharmacokinetics and pharmacodynamics of VKA leading to unpredictable anticoagulant response and difficulties in maintaining an adequate INR, these drugs are often underused, despite their undisputable clinical efficiency. Meanwhile, 


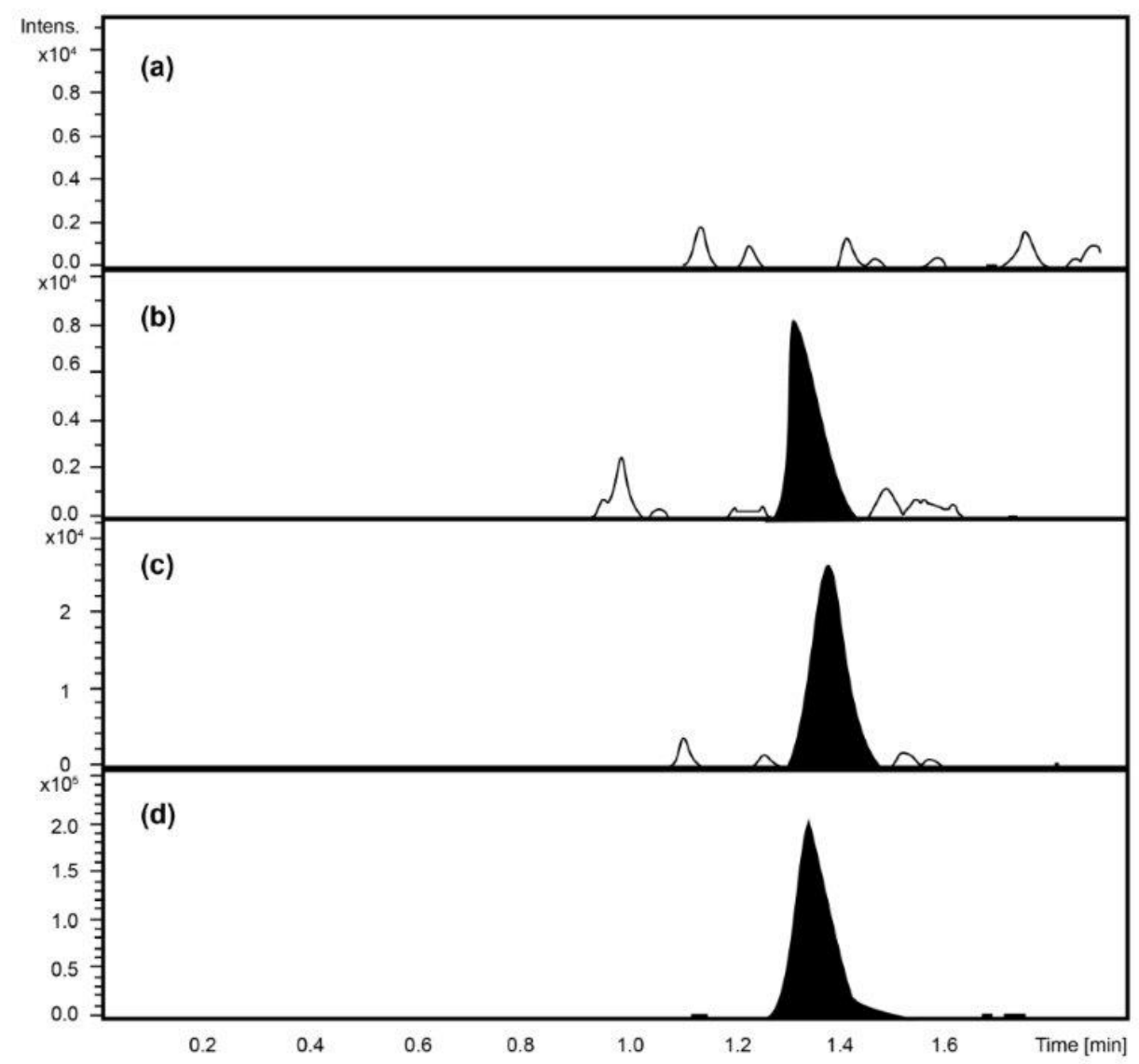

Figure 3. Chromatograms of: (a) blank plasma sample; (b) rivaroxaban at LLOQ, $24.00 \mathrm{ng} / \mathrm{mL}$; (c) rivaroxaban pre-dose - Sample_1, concentration found $88.40 \mathrm{ng} / \mathrm{ml}$; (d) rivaroxaban $3 \mathrm{hours}$ post-dose - Sample_2, concentration found $704.30 \mathrm{ng} / \mathrm{ml}$.

factors such as short half-life, few food and drug interactions, predictable pharmacokinetics and pharmacodynamics, wide therapeutic windows, and low genetic variability supported the use of NOACs, including rivaroxaban, without the need for routine laboratory monitoring [6]. A number of clinical settings have been established, however, laboratory testing to determine at least the presence or absence of anticoagulant effect in NOAC-treated patients may be critical. Since the anticoagulant response to NOACs does not seem to be completely immune to factors such as coexisting pathologies, drug and food interactions, treatment adherence and even genetic variability, periodic rather than "circumstantial" testing may be needed, at least in selected categories of patients [6].

Although less affected by altered kidney function than dabigatran, for which about $80 \%$ of the dose is eliminated via urine [16], rivaroxaban is not completely free of such interactions, as reflected by the lower drug doses used in patients with impaired kidney function in clinical trials $[3,17]$. However, whereas only about one third 
Table 2. Between-run accuracy (Bias), precision (CV) and recovery for rivaroxaban (CV - coefficient of variation, SD - standard deviation, $\mathrm{C}$ - concentration)

\begin{tabular}{ccccc}
\hline $\mathbf{c}_{\text {nominal }}(\mathbf{n g} / \mathbf{m l})$ & $\begin{array}{c}\text { Mean } \mathbf{c}_{\text {found }} \\
(\mathbf{n g} / \mathbf{m l}( \pm \mathbf{S D}))\end{array}$ & Bias $(\%)$ & $\mathbf{C V ~}(\%)$ & $\begin{array}{c}\text { Mean recovery } \\
(\% \mathbf{\%}) \mathbf{S D})\end{array}$ \\
\hline $24.00(\mathrm{LLOQ})$ & $24.94(3.26)$ & 3.9 & 13.1 & $106.2(8.7)$ \\
\hline $48.00(\mathrm{QCA})$ & $49.71(2.56)$ & 3.6 & 5.1 & $102.7(7.6)$ \\
\hline $192.00(\mathrm{QCB})$ & $189.09(16.50)$ & -1.5 & 8.7 & $103.4(5.9)$ \\
\hline $576.00(\mathrm{QCC})$ & $540.62(34.98)$ & -6.1 & 6.5 & $96.1(8.0)$ \\
\hline
\end{tabular}

of the rivaroxaban dose is eliminated unchanged by the kidneys, the remaining two thirds undergo conversion into inactive metabolites in the liver, via CYP3A4-dependent pathways [18]. Additionally, similarly to all the other NOACs, rivaroxaban absorption is highly dependent on the p-glycoprotein transporting system, hence rivaroxaban should not be combined with strong dual CYP3A4 and p-glycoprotein inhibitors or inducers. Isolated use of p-glycoprotein inhibitors/ inducers, however, does not seem to significantly affect rivaroxaban pharmacokinetics [19].

Although rivaroxaban appears to have low variability, pharmacokinetic studies reported high interindividual variations in drug plasma levels, even among apparently healthy individuals [20]. In patients with venous thromboembolism, both peak $(22 \mu \mathrm{g} / \mathrm{L}$ to $535 \mu \mathrm{g} / \mathrm{L})$ and trough $(6 \mu \mathrm{g} / \mathrm{L}$ to $239 \mu \mathrm{g} / \mathrm{L}$ ) rivaroxaban levels varied widely, although the clinical impact of these variations has not been reported yet [21]. These clinical and pharmacokinetic data support the critical role of rivaroxaban laboratory testing, at least in certain circumstances and/or in selected patients.

Although some of the classic coagulation tests have been validated for clinical use, mainly in emergency settings, these assays can generally provide only qualitative evaluation of the presence or absence of NOACs, and no correlation between test results and clinical outcomes has been established so far. Meanwhile, newer, more specific assays validated for clinical use have a number of drawbacks, including high costs, lack of wide availability, and complex, time-consuming protocols. Developing new laboratory techniques and/or adapting already existing methods will allow us to assess rapidly, accurately, and cost-efficiently the level of anticoagulation in rivaroxaban-treated patients.

Several methods have been described in the literature for the quantification of rivaroxaban from human plasma [8-12] or urine [10]. Kuhn et al. determined rivaroxaban and dabigatran levels in human plasma using the UPLC-MS/ MS method. The method uses upper pressure liquid chromatography (not widely available in comparison with HPLC) and the total run time of the method $(2.5 \mathrm{~min})$ is longer than in our developed assay [8]. Korostelev et al. measured dabigatran and rivaroxaban human plasma concentrations by a validated LC-MS/MS method. The calibration range was $2.5-500 \mathrm{ng} / \mathrm{mL}$ and total run time was $5 \mathrm{~min}$. However, besides quite long analysis time, the upper quantification limit of $500 \mathrm{ng} / \mathrm{mL}$ may not be appropriate for rivaroxaban quantification after multiple daily doses are administered to patients [9].

Cheng et al. developed a surface-assisted laser desorption/ionization mass spectrometry method to quantify rivaroxaban in human plasma and urine. Dispersive liquid-liquid microextraction was used for sample preparation. However, our 
method has the advantage of easier and shorter sample preparation which avoids use of toxic reagents such as tetrahydrofuran, chloroform and dimetylsulphoxide [10]. Rohde et al. developed an LC-MS/MS method and successfully applied it to several clinical studies of rivaroxaban [11]. The upper quantification limit was 500ng/ $\mathrm{mL}$, and the processed human plasma sample volumes were $0.2 \mathrm{~mL}$. Total run time for each injected sample was $5 \mathrm{~min}$. In comparison, our method uses smaller sample volumes $(0.1 \mathrm{~mL})$ and has considerably shorter run time (1.7 $\mathrm{min})$. Schellings et al. determined rivaroxaban and dabigatran human plasma levels in patients after major orthopedic surgery, the method having a total run time $4.75 \mathrm{~min}$ [12]. Srinivas Reddy et al. developed and validated a high throughput LC-MS/MS method for the quantification of rivaroxaban with a calibration range of 2-500 $\mathrm{ng} / \mathrm{mL}$ and a short run time of $2 \mathrm{~min}$; however, solid phase extraction was used for sample of plasma sample $(100 \mu \mathrm{L})$ and has a shorter run-time compared to most methods described in the literature, as well as simple, rapid and inexpensive sample preparation.

The method has been validated according to current international guidelines [14, 15]. The method showed good linearity, sensitivity (LLOQ of $24.00 \mathrm{ng} / \mathrm{mL}$ ), good accuracy and precision over the studied concentration range (Table I and Table II).

The developed high-throughput analytical method was successfully applied for therapeutic drug monitoring of hospitalized patients being under treatment with rivaroxaban (daily doses of $20 \mathrm{mg}$ ). A descriptive statistics of the rivaroxaban plasma levels before and 3 hours after drug administration is presented in Table III. As can be observed, the mean rivaroxaban plasma levels before administration of a new dose are about $123.3 \mathrm{ng} / \mathrm{mL}$, with an inter-subject variability of $51 \%$. The increase of rivaroxaban concentration

Table 3. Descriptive statistics of rivaroxaban plasma concentration, normalised concentration, and INR pre-dose and 3 hours after a new $20 \mathrm{mg}$ rivaroxaban dose administration $(\mathrm{n}=\mathbf{2 9})$

Sample type

\begin{tabular}{lcccccccc}
\hline & \multicolumn{4}{c}{$\begin{array}{c}\text { Pre-dose } \\
\text { (Number of samples, N= 29) }\end{array}$} & \multicolumn{3}{c}{$\begin{array}{c}\text { 3 hours post-dose } \\
\text { (Number of samples, N= 29) }\end{array}$} \\
\hline Parameter & Mean & SD & Median & CV\% & Mean & SD & Median & CV\% \\
\hline $\begin{array}{l}\text { Concentration } \\
\text { (ng/mL) }\end{array}$ & 123.33 & 63.10 & 113.00 & 51.16 & 533.93 & 201.27 & 514.20 & 37.70 \\
\hline $\begin{array}{l}\text { Normalized } \\
\text { concentration } \\
\text { (ng/mL/kg) }\end{array}$ & 1.37 & 0.72 & 1.20 & 52.64 & 6.28 & 3.24 & 5.50 & 51.60 \\
\hline INR & 1.16 & 0.11 & 1.10 & 9.70 & 1.53 & 0.28 & 1.50 & 18.07 \\
\hline
\end{tabular}

preparation, which greatly increases the cost of analysis and sample preparation time [13].

Our developed and validated method is rapid, simple and sensitive, requires small volumes after drug administration is about 4.3 times $(533.9 \mathrm{ng} / \mathrm{mL})$. The same trend and with similar magnitude can be observed for body weightnormalized concentration. The mean INR values 

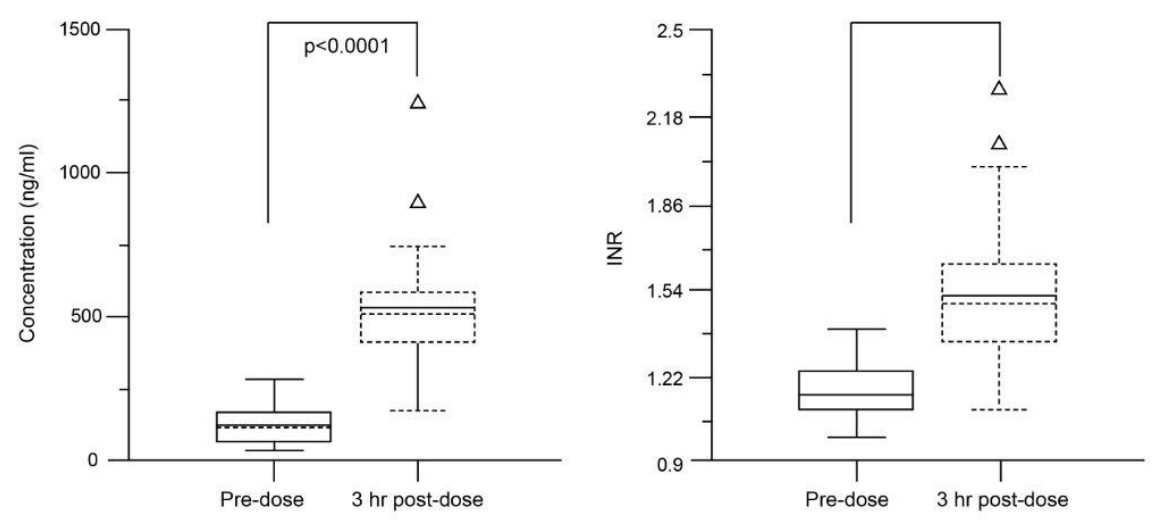

\section{Figure 4. Box-plot representations of rivaroxaban plasma levels pre-dose and 3 hours post-dose (left) and the corresponding variation of INR values (right).}

after drug administration (1.53) show an average increase by about $32 \%$ in comparison with the values before the new dose (1.16).

Box-plot representations (Figure 4) show the increase of rivaroxaban plasma levels before and 3 hours after drug administration and the effect on INR values, with their statistical significance (two-ways ANOVA with source of variation subject and Sample type - pre- or after dose, significance for $\mathrm{p}<0.05$ ).

In conclusion, a simple, rapid, inexpensive high throughput LC-MS/MS method for the determination of rivaroxaban in human plasma was developed, validated and used in therapeutic drug monitoring. When compared to other published assays, the presented analytical method is faster (short run times and simple, rapid sample preparation), which is essential in routine analysis with large numbers of samples, and has an adequate sensitivity for the proposed utilization. The method was successfully applied for determination of rivaroxaban plasma levels in hospitalized patients and can also be applied in pharmacokinetic studies.
Possible limitations during method transfer to a clinical laboratory include possible limitations related to the available infrastructure at the transfer site (e.g. the mass spectrometer characteristics, type, sensitivity, differences in liquid chromatographs), however, in this case the analytical method may be easily adapted to the new infrastructure environment and then revalidated. Another possible limitation is the cost per clinical sample analyzed in case of very low number of samples submitted for analysis, as in each analytical series at least one new calibration curve and control samples should be analyzed even if one only needs to analyze 2-3 samples.

\section{Acknowledgements}

This work was supported by an Internal Research Grant of University of Medicine and Pharmacy of Tîrgu Mureș, Romania, in partnership with SC Cosamext SRL Tîrgu Mureș, Romania. Research contract number 8688/22.07.2015.

\section{Conflict of Interest}

The authors declare that they have no conflict of interest. 


\section{Abbreviations}

$\begin{array}{ll}\text { APCI } & \text { atmospheric pressure chemical } \\ & \text { ionization } \\ = & \text { coefficient of variation } \\ \text { CV } & \text { electrospray ionisation } \\ \text { ESI } & \text { mC-MS/MS = liquid chromatography tandem } \\ & \text { mass spectrometry } \\ \text { LLOQ } & =\text { lower limit of quantification } \\ \text { MS/MS } & \text { tandem mass spectrometry } \\ \text { QC } & =\text { quality control } \\ \text { SPE } & =\text { solid phase extraction } \\ \text { UPLC-MS/ }= & \text { ultra performance liquid } \\ \text { MS } & \text { chromatography tandem mass } \\ & \text { spectrometry }\end{array}$

\section{References}

1. Mathers CD, Loncar D. Projections of global mortality and burden of disease from 2002 to 2030. PLoS Med. 2006 Nov; 3(11): e442. 2011-30.

2. Connolly SJ, Ezekowitz MD, Yusuf S, Eikelboom J, Oldgren J, Parekh A, et al. Dabigatran versus warfarin in patients with atrium fibrillation. N Engl J Med. 2009 Sep;361(12):1139-51. DOI: 10.1056/NEJMoa0905561

3. Patel MR, Mahaffey KW, Garg J, Pan G, Singer DE, Hacke W, et al. Rivaroxaban versus warfarin in nonvalvular atrial fibrilation. N Engl J Med. 2011 Sep; 365(10):883-91. DOI: 10.1056/NEJMoa1009638

4. Granger CB, Alexander JH, McMurray JJ, Lopes RD, Hylek EM, Hanna M, et al. Apixaban versus warfarin in patients with atrial fibrillation. N Engl J Med. 2011 Sep; 365(11):981-92. DOI: 10.1056/NEJMoa1107039

5. Giugliano RP, Ruff CT, Braunwald E, Murphy SA, Wiviott SD, Halperin JL, et al. Edoxaban versus warfarin in patients with atrial fibrillation. $\mathrm{N}$ Engl $\mathrm{J}$ Med. 2013 Nov; 369(22):2093-104. DOI: 10.1056/ NEJMoa1310907

6. Scridon A, Șerban RC. Laboratory monitoring - a turning point in the use of new oral anticoagulants. Ther Drug Monit. 2016 Feb; 38(1):12-21. DOI: 10.1097/ FTD.0000000000000247
7. Baglin T, Hillarp A, Tripodi A, Elalamy I, Buller H, Ageno W. Measuring oral direct inhibitors (ODIs) of thrombin and factor Xa: a recommendation from the subcommittee on control of anticoagulation of the scientific and standardisation committee of the international society on thrombosis and haemostasis. J Thromb Haemost. 2013 Jan;11:756-60. DOI: 10.1111/jth.12149

8. Kuhn J, Gripp T, Flieder T, Dittrich M, Hendig D, Busse J, et al. UPLC-MRM mass spectrometry method for measurement of the coagulation inhibitors dabigatran and rivaroxaban in human plasma and its comparison with functional assays. PLoS ONE. 2015 Dec; 10(12):e0145478. DOI: 10.1371/journal.pone.0145478

9. Korostelev M, Bihan K, Ferreol L, Tissot N, Hulot JS, Funck-Brentano C, et al. Simultaneous determination of rivaroxaban and dabigatran levels in human plasma by high-performance liquid chromatography-tandem mass spectrometry. J Pharm Biomed Anal. 2014 Nov;100:230-5. DOI: 10.1016/j.jpba.2014.08.011

10. Cheng YH, Chen WC, Chang SY. Rapid determination of rivaroxaban in human urine and serum using colloidal palladium surface-assisted laser desorption/ ionization mass spectrometry. Rapid Commun Mass Spectrom. 2015 Nov; 29(21):1977-83. DOI: 10.1002/ rcm.7308

11. Rohde G. Determination of rivaroxaban - a novel, oral, direct Factor Xa inhibitor - in human plasma by high-performance liquid chromatography-tandem mass spectrometry. J Chromatogr B Analyt Technol Biomed Life Sci. 2008 Sep; 872(1-2):43-50. DOI: 10.1016/j. jchromb.2008.07.015

12. Schellings MW, Boonen K, Schmitz EM, Jonkers F, van den Heuvel DJ, Besselaar A, et al. Determination of dabigatran and rivaroxaban by ultra-performance liquid chromatography-tandem mass spectrometry and coagulation assays after major orthopaedic surgery. Thromb Res. 2016 Mar; 139:128-34. DOI: 10.1016/j. thromres.2016.01.012

13. Srinivas Reddy G, Prasad Reddy SLN, Shiva Kumar Reddy L. Development and validation of Hplc-Ms/Ms Method for Rivaroxaban quantitation in human plasma using solid phase extraction procedure. Orient J Chem. 2016 Dec; 32(2):1145-54. DOI: $10.13005 /$ ojc/320240

14. U.S. Department of Health and Human Services. Food and Drug Administration. Center for Drug Evaluation and Research (CDER). Center for Veterinary Medicine (CVM). Guidance for Industry. Bioanalytical Method 
Validation. 2013 Sep; available at http://www.fda.gov/ downloads/drugs/ guidancecomplianceregulatoryinformation/guidances/ucm368107.pdf

15. European Medicines Agency. Committee for Medicinal Products for Human Use (CHMP). Guideline on bioanalytical method validation. 2011, Jul; available at http:// www.ema.europa.eu/docs/en_GB/document_library/ Scientific_guideline/2011/08/WC500109686. $\overline{\text { pdf }}$

16. Stangier J, Stähle H, Rathgen K, Fuhr R. Pharmacokinetics and pharmacodynamics of the direct oral thrombin inhibitor dabigatran in healthy elderly subjects. Clin Pharmacokinet. 2008 Jan; 47(1):47-59. DOI: $10.2165 / 00003088-200847010-00005$

17. EINSTEIN-PE Investigators, Büller HR, Prins MH, Lensin AW, Decousus H, et al. Oral rivaroxaban for the treatment of symptomatic pulmonary embolism. N Engl J Med. 2012 Apr; 366(14):1287-97. DOI: 10.1056/NEJMoa1113572

18. Perzborn E, Roehrig S, Straub A, Kubitza D, Misselwitz F. The discovery and development of rivaroxaban, an oral direct factor Xa inhibitor. Nat Rev Drug Discov. 2011 Jan;10(1):61-75. DOI: 10.1038/nrd3185

19. Gnoth MJ, Buetehorn U, Muenster U, Schwartz T, Sandmann S. In vitro and in vivo P-glycoprotein transport characteristics of rivaroxaban. J Pharmacol Exp Ther. 2011 Jul;338(1):372-80. DOI: 10.1124/ jpet.111.180240

20. Mueck W, Becka M, Kubitza D, Voith B, Zuehlsdorf M. Population model of the pharmacokinetics and pharmacodynamics of rivaroxaban - an oral, direct factor Xa inhibitor-in healthy subjects. Int J Clin Pharmacol Ther. 2007 Jun; 45(6):335-44. DOI: 10.5414/CPP45335

21. Ten Cate H. New oral anticoagulants: discussion on monitoring and adherence should start now! Thromb J 2013 Jun; 11(1):8. DOI: 10.1186/1477-9560-11-8 